\title{
Controle de Egeria densa E Egeria najas EM CAIXA-D’ÁGUA UTILIZANDo o HeRBICIDA DIQUAT ${ }^{1}$
}

\author{
Control of Egeria densa and Egeria najas in Water Tanks Using Diquat
}

MARTINS, D. ${ }^{2}$,VELINI, E.D. ${ }^{3}$ e NEGRISOLI, E. ${ }^{4}$

\begin{abstract}
RESUMO - Este estudo foi realizado no Núcleo de Pesquisas Avançadas em Matologia da FCA/UNESP, campus de Botucatu-SP, com o objetivo de avaliar o controle de Egeria densa e Egeria najas, utilizando o herbicida diquat. As doses do herbicida para ambas as espécies foram: 0,$1 ; 0,25 ; 0,5 ; 1,0 ;$ e $1,5 \mathrm{ppm}$, além de uma testemunha sem aplicação do herbicida. As parcelas experimentais foram constituídas por caixas-d'água de 0,60 $\mathrm{m}$ de diâmetro por 1,10 m de profundidade, contendo 300 litros de água, as quais foram colocadas a pleno sol, com 15 plantas de cada espécie. A aplicação ocorreu com as plantas apresentando $70 \mathrm{~cm}$ de comprimento. O controle foi avaliado visualmente aos $5,7,10,14,26,31,38$ e 45 dias após a aplicação dos herbicidas, por meio de uma escala percentual de notas, em que zero correspondia a nenhum controle e $100 \%$ ao controle total das plantas. Para Egeria najas, $0,5 \mathrm{ppm}$ de diquat foi o suficiente para determinar controle total das plantas, e não ocorreram rebrotas posteriormente. Nas plantas de Egeria densa ocorreram rebrotas a partir dos 45 dias após a aplicação do herbicida diquat, com maior intensidade nas doses menores; uma reaplicação de 0,5 ppm determinou também a morte completa das plantas.
\end{abstract}

Palavras-chave: herbicida, planta imersa, planta aquática.

ABSTRACT - The aim of this research was to evaluate the control of Egeria densa and Egeria najas, using the herbicide diquat. The herbicide doses used for both species were: 0.1; 0.25; 0.5 ; 1.0; and 1.5ppm. Tanks with $0.60 \times 1.10 \mathrm{~m}$ dimension constituted the plots. Visual evaluation at $5,7,10,14,26,31,38$, and 45 days after herbicide application was performed through percentage score scale, with 100\% corresponding to total plant control. For Egeria najas, $0.5 \mathrm{ppm}$ of diquat was sufficient to determine total plant control, with no posterior occurrence of sprouts. For Egeria densa, sprouts occurred 45 days after herbicide application with highest intensity at the lowest doses. Reapplication doses of 0.5ppm also determined death of the plants.

Key words: herbicide, immersed plant, aquatic plant.

\section{INTRODUÇÃO}

As plantas daninhas aquáticas normalmente se tornam problemáticas quando o homem altera seu habitat original. Em condições naturais, há fatores como luz, nutrientes, espaço e os inimigos naturais (insetos e doenças) atuando na flutuação dessas populações, mantendo-as abaixo do nível de dano econômico.

Dentre as principais plantas daninhas aquáticas, podem-se destacar: Eichhornia crassipes, Pistia stratiotes, Echinochloa polystachya, Brachiaria subquadripara, Typha dominguensis, Egeria densa, Egeria najas e

Recebido para publicação em 12.1.2005 e na forma revisada em 24.3.2005.

2 Prof Livre Docente, Departamento de Produção Vegetal, FCA/UNESP, Caixa Postal 237, $18603-970$ Botucatu-SP, $<$ dmartins@fca.unesp.br>; ${ }^{3}$ Prof Dr., Departamento de Produção Vegetal, FCA/UNESP; ${ }^{4}$ Eng.-Agr., Doutorando em Agricultura do Departamento de Produção Vegetal, FCA/UNESP. 
espécies aquáticas dos gêneros Polygonum, Salvinia e Cyperus (Martins et al., 2002).

A presença excessiva de plantas aquáticas tem prejudicado a utilização da água para navegação, recreação e esportes náuticos, geração de energia em usinas hidrelétricas e a captação de água para irrigação e consumo humano e animal, no Brasil e em diversos países (Burkhalter, 1975; Pitelli, 1998). No Brasil, o controle de plantas aquáticas submersas tornou-se alvo de estudos intensos nos últimos anos, devido aos prejuízos provocados por esse tipo de vegetação nos reservatórios de usinas hidrelétricas (Tanaka et al., 2002; Negrisoli et al., 2003; Carvalho et al., 2003; Corrêa, 2004; Velini et al., 2004).

Em águas transparentes e com sedimentos ricos em nutrientes ocorre grande proliferação de plantas daninhas imersas, tanto ancoradas como flutuantes na coluna d'água. Nesse ambiente, é comum a ocorrência de plantas como Egeria najas, Egeria densa e Ceratophyllum demersum, as quais são de grande importância em reservatórios utilizados na produção de energia elétrica, principalmente pelos danos causados no processo de geração de energia.

Dentre os possiveis manejos desse tipo de planta daninha aquática - plantas imersas (E. densa e E. najas) - que visam reduzir o seu desenvolvimento ou mesmo a sua eliminação, tem-se o controle químico, o qual tem sido amplamente estudado com outras espécies, principalmente no Brasil (Cardoso, 2001; Tanaka et al., 2002b; Martins et al., 2002b; Marcondes et al., 2003; Cardoso et al., 2004).

O objetivo deste trabalho foi avaliar a eficiência de controle proporcionado por diferentes doses do herbicida diquat sobre as plantas de E. densa e E. najas, em caixa-d'água.

\section{MATERIAL E MÉTODOS}

O experimento foi instalado e conduzido no Núcleo de Pesquisas Avançadas em Matologia (NUPAM) do Departamento de Produção Vegetal da Faculdade de Ciências Agronômicas - UNESP, campus de Botucatu/ SP.

As plantas de Egeria densa e Egeria najas foram cultivadas em caixas de fibra de vidro com 0,60 m de diâmetro por $1,1 \mathrm{~m}$ de profundidade, contendo um volume de 300 litros de água. Os fragmentos apicais das espécies estudadas foram coletados no reservatório de Jupiá, no município de Itapura-SP; estas espécies se encontravam em estádio adulto de desenvolvimento. Em cada caixa foram colocados $20 \mathrm{~cm}$ de substrato, sendo solo de várzea na parte infeior $(15 \mathrm{~cm})$ e areia nos $5 \mathrm{~cm}$ superiores .

Foram plantados 15 fragmentos de cada espécie por caixa, e cada muda apresentava $0,2 \mathrm{~m}$ de comprimento. As plantas foram deixadas crescer por 140 dias e, após atingirem em torno de 0,7 m de comprimento, foi iniciado o estudo. Os tratamentos e doses utilizados nos estudos com $E$. densa e $E$. najas foram: diquat (Reward $240 \mathrm{~g} \mathrm{~L}^{-1}$ ) a 0,$10 ; 0,25 ; 0,5 ; 1,0$; e $1,5 \mathrm{ppm}$, além de uma testemunha sem aplicação de herbicida.

Os tratamentos foram dispostos no delineamento experimental em blocos ao acaso, com seis repetições. As doses do herbicida diquat foram previamente preparadas em laboratório com auxílio de balões volumétricos e, por meio de uma proveta, foram adicionadas às caixas-d'água. Houve duas épocas de aplicação do herbicida diquat: a primeira no dia 17/09/1999 e a segunda no dia 01/11/1999, 45 dias após a primeira aplicação, apenas para as caixas-d'água que receberam os tratamentos de 0,5, 1,0 e 1,5 ppm de diquat no estudo com Egeria densa.

As avaliações de controle foram efetuadas de forma visual aos 5, 7, 10, 14, 26, 31, 38 e 45 dias após a aplicação do herbicida, para a primeira época de aplicação e, para a segunda, aos $4,8,14,16,22$ e 28 , por meio de uma escala percentual de notas, na qual zero consistiu em nenhum controle e $100 \%$ em controle total das plantas. As avaliações foram feitas até o desaparecimento ou estabilização dos sintomas (Sociedade Brasileira da Ciência das Plantas Daninhas, 1995).

Os parâmetros utilizados para o estabelecimento das notas foram: quantidade de plantas mortas, acúmulo de biomassa, inibição do crescimento, quantidade e uniformidade das injúrias e capacidade de rebrota das plantas.

A análise de variância foi efetuada pelo teste "F" e as médias dos tratamentos 
foram comparados pelo teste " $\mathrm{t}$ " a $5 \%$ de probabilidade.

\section{RESULTADOS E DISCUSSÃO}

\section{Egeria densa}

Na Tabela 1 estão apresentados os resultados da porcentagem de controle de plantas de E. densa em diferentes épocas de avaliação, após a aplicação do herbicida diquat.

Nota-se, aos cinco dias após a aplicação das diferentes doses do herbicida diquat, que nenhuma das doses proporcionou controle satisfatório, porém nas doses maiores ou iguais a $0,5 \mathrm{ppm}$ os efeitos de intoxicação nas plantas de E. densa foram mais severos. Aos sete dias, verificou-se incremento no controle da planta em estudo, mas ainda insatisfatório, sendo o mesmo observado também aos dez dias.

Observa-se, aos 14 dias após a aplicação das diferentes doses de diquat, que as caixasd'água que receberam as doses de 0,5, 1,0 e $1,5 \mathrm{ppm}$ de diquat apresentaram controles considerados bons, tendo sido semelhantes entre si. Já aos 26 dias as duas menores doses de diquat apresentaram decréscimo no controle de $E$. densa e as doses maiores de $0,5,1,0$ e $1,5 \mathrm{ppm}$ atingiram controle considerado excelente. $\mathrm{O}$ mesmo fato foi observado nas avaliações realizadas aos 31 e 38 dias após a aplicação do herbicida diquat, em que as doses menores proporcionaram controle insatisfatório e as maiores continuaram demonstrando controle excelente sobre a planta em estudo.

Aos 45 dias, ocasião da última avaliação e término previsto para o experimento, as doses de 0,1 e 0,25 ppm de diquat não proporcionavam mais nenhuma injúria visual e aparente sobre as plantas de E. densa, e as doses de 0,5, 1,0 e 1,5 ppm apresentaram pequenas rebrotas em algumas plantas, em menor quantidade nas doses maiores, evidenciando uma recuperação parcial delas, todavia, ainda, com o controle considerado bom ou excelente. Essas rebrotas determinaram reaplicações nas caixas-d'água que receberam as doses maiores de diquat, e os resultados desse novo ciclo de controle encontram-se na Tabela 2.

Na Tabela 2 estão apresentados os resultados da porcentagem de controle sobre as plantas de E. densa remanescentes da primeira fase do estudo, em diferentes épocas de avaliação, após a reaplicação das doses de $0,5,1,0$ e 1,5 ppm de diquat. Verifica-se que o controle proporcionado pelas diferentes doses de diquat foi bom ou excelente somente a partir dos 14 dias após a aplicação, como observado também na primeira fase do estudo, mantendo-se elevados até os 28 dias, quando do encerramento definitivo do experimento, com $100 \%$ de controle. Também Martins et al. (2002b), ao estudarem o herbicida diquat, constataram que as doses testadas $(460,960$ e $1.400 \mathrm{~g}$ i.a. ha $\mathrm{h}^{-1}$ ) foram eficientes no controle

Tabela 1 - Porcentagem de controle de Egeria densa em diferentes períodos após a aplicação do herbicida diquat. Botucatu-SP

\begin{tabular}{|c|c|c|c|c|c|c|c|c|c|}
\hline \multirow{2}{*}{ Tratamento } & \multirow{2}{*}{$\begin{array}{c}\text { Dose } \\
(\mathrm{ppm})\end{array}$} & \multicolumn{8}{|c|}{ Dias após aplicação } \\
\hline & & 05 & 07 & 10 & 14 & 26 & 31 & 38 & 45 \\
\hline 1. diquat & 0,1 & $2,5 \mathrm{c}$ & $5,0 \mathrm{c}$ & $8,5 \mathrm{~d}$ & $9,0 \mathrm{c}$ & $6,5 \mathrm{a}$ & $14,3 \mathrm{~b}$ & $4,3 \mathrm{c}$ & $0,0 \mathrm{c}$ \\
\hline 2. diquat & 0,25 & $17,5 \mathrm{~b}$ & $18,3 \mathrm{~b}$ & $20,0 \mathrm{c}$ & $24,2 \mathrm{~b}$ & $19,2 \mathrm{c}$ & $5,5 \mathrm{~b}$ & $9,6 \mathrm{~b}$ & $0,0 \mathrm{c}$ \\
\hline 3. diquat & 0,5 & $47,2 \mathrm{a}$ & $55,0 \mathrm{a}$ & $58,7 \mathrm{a}$ & $95,2 \mathrm{a}$ & $98,0 \mathrm{a}$ & $100,0 \mathrm{a}$ & $100,0 \mathrm{a}$ & $94,1 \mathrm{a}^{1 /}$ \\
\hline 4. diquat & 1,0 & $41,5 \mathrm{a}$ & $49,2 \mathrm{a}$ & $51,7 \mathrm{~b}$ & 96,5 a & $98,0 \mathrm{a}$ & $100,0 \mathrm{a}$ & $100,0 \mathrm{a}$ & $92,8 \mathrm{ab}^{\underline{1}}$ \\
\hline 5. diquat & 1,5 & $44,2 \mathrm{a}$ & $49,7 \mathrm{a}$ & $56,2 \mathrm{a}$ & $96,2 \mathrm{a}$ & $96,5 \mathrm{a}$ & $100,0 \mathrm{a}$ & $100,0 \mathrm{a}$ & $91,6 \mathrm{~b}^{1 /}$ \\
\hline 6. Testemunha & - & - & - & - & - & - & - & - & - \\
\hline F tratamento & & $44,21 * *$ & $89,63 * *$ & $124,17 * *$ & $3.029,13 * *$ & $9.282,03 * *$ & $12.289,55^{* *}$ & $61.537,63^{* *}$ & $5.149,96^{* *}$ \\
\hline F bloco & & $1,96^{\mathrm{ns}}$ & $1,71^{\mathrm{ns}}$ & $0,56^{\mathrm{ns}}$ & $1,05^{\mathrm{ns}}$ & $2,25^{\text {ns }}$ & $1,17^{\mathrm{ns}}$ & $1,26^{\mathrm{ns}}$ & $1,64^{\text {ns }}$ \\
\hline CV $(\%)$ & & 23,6 & 16,3 & 13,0 & 3,0 & 1,9 & 1,7 & 0,8 & 3,1 \\
\hline DMS & & 8,78 & 6,95 & 6,11 & 2,35 & 1,43 & 1,32 & 0,60 & 2,09 \\
\hline
\end{tabular}

$\underline{1}$ tratamentos com rebrotas. $* *$ significativo a $1 \%$ de probabilidade. ${ }^{\text {ns }}$ não-significativo.

Médias seguidas de mesma letra na coluna não diferem estatisticamente entre si pelo teste $t(P>0,05)$. 
de Salvinia molesta, Pistia stratiotes e Eichhornia crassipes.

Ressalta-se que, no encerramento do experimento, esvaziaram-se as caixas-d'água, e as mortes definitivas das plantas de E. densa foram atestadas através de minuciosa avaliação das plantas que estavam decompostas no fundo da caixa, com revolvimento inclusive do substrato, para analisar o sistema radicular.

Portanto, as doses de 0,1 e 0,25 ppm de diquat foram ineficientes no controle de E. densa, e as doses de 0,5, 1,0 e 1,5 ppm foram eficientes, porém ocorreram rebrotas de plantas, com maior intensidade nas doses menores. As rebrotas das plantas de E. densa foram controladas de forma eficiente com uma reaplicação do herbicida diquat; a menor dose de diquat reaplicada de $0,5 \mathrm{ppm}$ foi o suficiente para determinar a morte completa das plantas de E. densa.

\section{Egeria najas}

Na Tabela 3 estão apresentados os resultados da porcentagem de controle de plantas de E. najas em diferentes épocas de avaliação, após a aplicação do herbicida diquat.

Nota-se, aos cinco dias após a aplicação das diferentes doses do herbicida diquat, que nenhuma das doses testadas proporcionou controle satisfatório, porém nas doses maiores ou iguais a $0,5 \mathrm{ppm}$ os efeitos de intoxicação nas plantas de E. najas foram mais severos. Aos sete dias, verificou-se incremento no controle da planta em estudo, porém ainda insatisfatório, sendo o mesmo observado também aos dez dias.

Tabela 2 - Porcentagem de controle de Egeria densa em diferentes períodos após a reaplicação do herbicida diquat. Botucatu-SP

\begin{tabular}{|c|c|c|c|c|c|c|c|}
\hline \multirow{2}{*}{ Tratamento } & \multirow{2}{*}{$\begin{array}{c}\text { Dose } \\
\text { (ppm) }\end{array}$} & \multicolumn{6}{|c|}{ Dias após reaplicação } \\
\hline & & 04 & 08 & 14 & 16 & 22 & 28 \\
\hline 1. diquat & 0,5 & $6,5 \mathrm{c}$ & $41,5 \mathrm{c}$ & $88,0 \mathrm{c}$ & $90,0 \mathrm{~b}$ & $94,5 \mathrm{c}$ & 100,0 \\
\hline 2. diquat & 1,0 & $12,5 \mathrm{~b}$ & $57,5 \mathrm{~b}$ & $89,5 \mathrm{~b}$ & $90,2 \mathrm{~b}$ & $95,0 \mathrm{~b}$ & 100,0 \\
\hline 3. diquat & 1,5 & $22,5 \mathrm{a}$ & $70,0 \mathrm{a}$ & $98,0 \mathrm{a}$ & $99,0 \mathrm{a}$ & $99,0 \mathrm{a}$ & 100,0 \\
\hline Testemunha & - & - & - & - & - & - & - \\
\hline F tratamento & & $51,58 * *$ & $112,34 * *$ & $581,67 * *$ & 626,34 & $365,00 * *$ & - \\
\hline F bloco & & $0,01^{\text {ns }}$ & $0,04^{\mathrm{ns}}$ & $3,00^{\mathrm{ns}}$ & $2,32^{\mathrm{ns}}$ & $1,00^{\mathrm{ns}}$ & - \\
\hline CV $(\%)$ & & 19,9 & 5,9 & 0,6 & 1,4 & 0,3 & $\therefore$ \\
\hline $\mathrm{DMS}$ & & 3,54 & 0,70 & 0,70 & 0,92 & 0,41 & - \\
\hline
\end{tabular}

** significativo a $1 \%$ de probabilidade. ${ }^{\text {ns }}$ não-significativo.

Médias seguidas de mesma letra na coluna não diferem estatisticamente entre si pelo teste $t(P>0,05)$.

Tabela 3 - Porcentagem de controle de Egeria najas em diferentes períodos após a aplicação do herbicida diquat. Botucatu-SP

\begin{tabular}{|c|c|c|c|c|c|c|c|c|c|}
\hline \multirow{2}{*}{ Tratamento } & \multirow{2}{*}{$\begin{array}{l}\text { Dose } \\
\text { (ppm) }\end{array}$} & \multicolumn{8}{|c|}{ Dias após aplicação } \\
\hline & & 05 & 07 & 10 & 14 & 26 & 31 & 38 & 45 \\
\hline 1. diquat & 0,1 & $2,5 \mathrm{c}$ & $5,0 \mathrm{c}$ & $8,5 \mathrm{~d}$ & $9,0 \mathrm{c}$ & $6,5 \mathrm{~d}$ & $5,5 \mathrm{c}$ & $4,3 \mathrm{c}$ & 0,0 \\
\hline 2. diquat & 0,25 & $17,5 \mathrm{~b}$ & $18,3 \mathrm{~b}$ & $20,0 \mathrm{c}$ & $24,1 \mathrm{~b}$ & $19,1 \mathrm{c}$ & $9,8 \mathrm{~b}$ & $7,1 \mathrm{~b}$ & 0,0 \\
\hline 3. diquat & 0,5 & $47,1 \mathrm{a}$ & $55,0 \mathrm{a}$ & $58,6 \mathrm{a}$ & $95,1 \mathrm{a}$ & $98,0 \mathrm{a}$ & $100,0 \mathrm{a}$ & $100,0 \mathrm{a}$ & 100,0 \\
\hline 4. diquat & 1,0 & $41,5 \mathrm{a}$ & $49,1 \mathrm{a}$ & $51,6 \mathrm{~b}$ & $96,5 \mathrm{a}$ & $98,0 \mathrm{a}$ & $100,0 \mathrm{a}$ & $100,0 \mathrm{a}$ & 100,0 \\
\hline 5. diquat & 1,5 & $44,1 \mathrm{a}$ & $49,6 \mathrm{a}$ & $56,1 \mathrm{ab}$ & $96,1 \mathrm{a}$ & $96,5 \mathrm{a}$ & $100,0 \mathrm{a}$ & $100,0 \mathrm{a}$ & 100,0 \\
\hline 6. Testemunha & - & - & - & - & - & - & - & - & - \\
\hline $\mathrm{F}$ tratamento & & $44,21 * *$ & 89,63 ** & $124,17 * *$ & $3.029,13 * *$ & $9.282,03 * *$ & $40.069,26^{* *}$ & $42.845,18^{* *}$ & - \\
\hline F bloco & & $1,96^{\text {ns }}$ & $1,71^{\mathrm{ns}}$ & $0,56^{\mathrm{ns}}$ & $1,05^{\mathrm{ns}}$ & $2,25^{\mathrm{ns}}$ & $0,35^{\text {ns }}$ & $0,38^{\text {ns }}$ & - \\
\hline CV $(\%)$ & & 23,6 & 16,3 & 13,0 & 3,0 & 1,9 & 1,0 & 1,0 & - \\
\hline DMS & & 8,70 & 6,95 & 6,12 & 2,34 & 1,43 & 0,75 & 0,74 & - \\
\hline
\end{tabular}

** significativo a $1 \%$ de probabilidade. ${ }^{\text {ns }}$ não-significativo.

Médias seguidas de mesma letra na coluna não diferem estatisticamente entre si pelo teste $\mathrm{t}(\mathrm{P}>0,05)$. 
Observa-se aos 14 dias após a aplicação das diferentes doses de diquat que as caixasd'água que receberam as doses de 0,5, 1,0 e $1,5 \mathrm{ppm}$ de diquat apresentaram controle considerado bom, tendo sido semelhantes entre si. Já aos 26 dias, as duas menores doses de diquat testadas mostraram decréscimo no controle de E. najas e as doses maiores de 0,5, 1,0 e $1,5 \mathrm{ppm}$ atingiram controle considerado excelente. O mesmo fato foi observado nas avaliações realizadas aos 31 e 38 dias após a aplicação do herbicida diquat, em que as doses menores proporcionaram controle insatisfatório e as maiores continuaram apresentando controle excelente sobre a planta em estudo, com $100 \%$ de controle e sem rebrota de plantas. Esses resultados foram semelhantes aos obtidos por Thayer \& Haller (1985), os quais constataram excelente controle na aplicação do herbicida nas espécies Spirodela polyrhiza, Pistia stratiotes e Salvinia rotundifolia.

Aos 45 dias, ocasião da última avaliação e término previsto para o experimento, as doses de 0,1 e 0,25 ppm de diquat não proporcionavam mais nenhuma injúria visual e aparente sobre as plantas de E. najas, e as de $0,5,1,0$ e 1,5 ppm mantiveram o controle total das plantas, sem rebrotas. Ressalta-se que, no encerramento do experimento, foram esvaziadas as caixas-d'água e analisaram-se as plantas de E. najas para confirmação da morte definitiva.

\section{LITERATURA CITADA}

BURKHALTER, A. P. The State of Florida aquatic weed control program. In: SIMPOSIUM ON WATER QUALITY AND MANAGEMENTE THROUGH BIOLOGICAL CONTROL, 1975, Gainesville. Proceedings... Gainesville: 1975. p. $15-19$.

CARDOSO, R. C. Sensibilidade de diferentes acessos de aguapé coletados em reservatórios do Estado de São Paulo a herbicidas. 2001. 95 f. Dissertação (Mestrado em Agricultura) - Universidade Estadual Paulista, Botucatu, 2001.

CARDOSO, L. R. et al. Variabilidade genética entre populações de Pistia stratiotes L. In: CONGRESSO BRASILEIRO DA CIÊNCIA DAS PLANTAS DANINHAS, 24., 2004, São Pedro. Anais... São Pedro: SBCPD, 2004. 107 p.
CARVALHO, F. T. et al. Influência da turbidez da água do rio Tietê na ocorrência de plantas aquáticas. Planta Daninha, v. 20, p. 63-73, 2003. (Edição especial)

CORREA, M. R.; VELINI, E. D.; BRAVIN, L. F. N. Utilização de GPS para monitoramento de infestação de macrófitas aquáticas em reservatórios de hidrelétricas. In: CONGRESSO BRASILEIRO DA CIÊNCIA DAS PLANTAS DANINHAS, 24., 2004, São Pedro. Anais... São Pedro: SBCPD, 2004. 108 p.

MARCONDES, D. A. S. et al. Eficiência de fluridone no controle de plantas daninhas aquáticas submersas e efeito sobre algumas características ambientais. Planta Daninha, v. 20, p. 63-73, 2003. (Edição especial)

MARTINS, D. et al. Resposta ao controle químico em acessos de Egeria najas coletadas em reservatórios de água no estado de São Paulo. In: CONGRESSO BRASILEIRO DA CIÊNCIA DAS PLANTAS DANINHAS, 23., 2002, Gramado. Anais... Gramado: SBCPD, 2002. 595 p.

MARTINS, D. et al. Controle químico de Pistia stratiotes, Eichhornia crassipes e Salvinia molesta em condições de caixa d'água. Planta Daninha, v. 20, p. 83-88, 2002 b. (Edição especial)

NEGRISOLI, E. et al. Degradação de diquat em condições de caixa d'água com e sem plantas de egéria. Planta Daninha, v. 21, p. 93-100, 2003. (Edição especial)

PITELLI, R. A. Macrófitas aquáticas no Brasil, na condição de problemáticas. In: WORKSHOP CONTROLE DE PLANTAS AQUÁTICAS, 1998, Brasília. Resumos... Brasília: Instituto Brasileiro de Meio Ambiente e dos Recursos Naturais Renováveis, 1998. p. 12-15.

SOCIEDADE BRASILEIRA DA CIÊNCIA DAS PLANTAS DANINHAS. Procedimentos para instalação, avaliação e análise de experimentos com herbicidas. Londrina: SBCPD, 1995. $42 \mathrm{p}$.

TANAKA, R. H. et al. Avaliação de herbicidas para o controle de Egéria em laboratório, caixa-d'água e represa sem fluxo de água. Planta Daninha, v. 20, p. 73-83, 2002. (Edição especial)

TANAKA, R. H. et al. Ocorrência de plantas aquáticas nos reservatórios da Companhia Energética de São Paulo. Planta Daninha, v. 20, p. 100-111, 2002b. (Edição especial)

THAYER, D. D.; HALLER, W. T. Effect of herbicides on floating aquatic plants. Jornal Aquatic Plant Manage., v. 23, p. 94-95, 1985.

VELINI, E. D. et al. Avaliação operacional do controle mecânico de macrófitas aquáticas imersas. In: CONGRESSO BRASILEIRO DA CIÊNCIA DAS PLANTAS DANINHAS, 24., 2004, São Pedro. Anais... São Pedro: SBCPD, 2004. 107 p.

Planta Daninha, Viçosa-MG, v. 23, n. 2, p. 381-385, 2005 